\title{
Numerical investigation on the generation, mixing and convection of entropic and compositional waves in a flow duct
}

\author{
Jocelino Rodrigues $^{\mathrm{a}, 1, *}$, Andrea Busseti ${ }^{\mathrm{b}, 2}$, Simone Hochgreb ${ }^{\mathrm{a}, 3}$ \\ ${ }^{a}$ University of Cambridge, Department of Engineering, Trumpington St, Cambridge CB2 1PZ, UK \\ ${ }^{b}$ Università di Genova, DIME, Via Montallegro 1, I-16145 Genoa, Italy
}

\begin{abstract}
Recent models and experiments have demonstrated that indirect noise can be generated via acceleration of either entropic or compositional perturbations through a nozzle. These studies found that the acoustic signature depends on the extent of the dispersion effects on the perturbation waves during the convection process. In this work, numerical simulations are undertaken using the URANS formulation to model the mixing of unsteady entropic and compositional waves in an open-ended flow duct in the transitional regime $(2500<R e<8100)$. The flow is perturbed by either heat addition or radial injection of an inert gas that is heavier (argon) or lighter (helium) than air. The computed temperature and mass fraction fields are compared to experimental results obtained using both intrusive and non-intrusive techniques. Agreement with the experimental data is good. The signatures of both perturbations are well captured using two-equation turbulence models. However, they lag behind the experimental results at downstream probe locations, suggesting that the centreline velocity is underpredicted. Dispersion effects on the compositional waves are shown to be insignificant for the system studied here. In the heat addition case, heat loss is found to be a key factor in capturing the correct entropic perturbation for long convection lengths.
\end{abstract}

Keywords: entropy waves; compositional waves; thermoacoustics; indirect noise.

${ }^{*}$ Corresponding author

Email address: jr699@cam.ac.uk (Jocelino Rodrigues)

${ }^{1} \mathrm{PhD}$ candidate.

${ }^{2}$ Visiting Master of Science (MSc) student.

${ }^{3}$ Professor of Experimental Combustion.

Preprint submitted to Journal of Sound and Vibration

February 10, 2020 


\section{Introduction}

Pressure fluctuations associated with combustion are often linked to either unsteady heat release (direct noise) or the acceleration of temperature, vorticity or composition non-uniformities (indirect noise) [1]. These acoustic disturbances can propagate back into the chamber and trigger combustion instabilities [2, 3]. Compositional fluctuations have recently been shown to be a significant contributor of combustion noise $[4,5]$. In practical devices, these arise due to incomplete mixing during the combustion process (stratification) or quenching of combustion products.

Separating the contributions of direct and indirect noise in gas turbines is difcases generated significant interest in the field leading to numerous numerical investigations. These generally employed either compressible Unsteady Reynolds-Averaged Navier-Stokes (URANS) with a rotationally symmetric geometry [19, 20, 21] or 
Large Eddy Simulation (LES) using 2D axisymmetric and/or fully 3D geometries 40 [22, 23, 24, 25, 26]. Models for heat addition varied from a simple volumetric energy source term (block heat deposition into a single volume), to more complex approaches, such as time-delayed activation (capturing the heat addition from each wire). The amount of energy added was adjusted to match experimental measurements of temperature and pressure fluctuations. No experimental validation of the dispersion of the convecting entropic wave was presented since the temperature signal for the entropic spot was measured at only one location downstream of the heating grid [26].

Ron and Chana [14] investigated the mixing of hot and cold streams in the Oxford Turbine Research Facility (OTRF), including time-resolved pressure measurements and spatially resolved temperature measurements. Simulation results (using 3D URANS and LES) of pressure spectra upstream of the throat were compared with experimental values. Measured and predicted noise results were of the same order of magnitude, but failed to predict the expected additional peaks due to entropic fluctuations. The evolution of compositional and entropic fluctuations in a realistic RQL and conditions studied, entropic noise was found to exceed compositional noise. No comparison with experimental data was presented.

Other work has specifically focused on the effects of convection (without acoustics) on the entropic waves using high-fidelity simulations. Original work by Sattelmayer [27] hypothesised that temperature fluctuations would decay by shear dispersion and turbulent mixing. This idea was further studied by Morgans et al. [28] and Giusti et al. [29]. Morgans et al. [28] suggested that the dispersion in a combustor may not be sufficiently fast and that entropic waves could contribute to acoustic oscillations. Giusti et al. [29] demonstrated how these entropic spots advect as a function of the non-dimensional Strouhal number (termed 'local Helmholtz number' in their study), demonstrating that the dispersion could simply be accounted for by the velocity profile obtained from URANS simulations.

In this particular study, gaseous species are injected as an unsteady Jet in Cross Flow (JICF); hence, prior work in this field is of interest.The fluid mechanics phenomena due to JICFs has been exhaustively studied, both experimentally and numerically. Experiments by Fric and Roshko [30] showed that four principal coherent vortical structures are present in cross-flow injection: jet shear-layer vortices, counter-rotating vortex pair, wake vortices, and a system of horseshoe vortices. The first attempt at studying the unsteadiness of JICFs using three dimensional URANS was made by $\mathrm{Hsu}$ et al. [31], where the standard $k-\varepsilon$ model with appropriate wall functions was used [32]. It was shown that three of the features described by Fric 
and Roshko [30], with the exception of the horseshoe vortices, could be captured by URANS when a JICF is modelled under fully unsteady 3D conditions. Innumerable studies have used numerical tools to understand transverse mixing jets, comparing results to experimental data (e.g. [33, 34, 35]). Although the collective interaction of small vortices make a very important contribution to the overall mixing process in jet in crossflow, URANS models are able to produce sensible results as long as an adequate turbulence model is chosen and a sufficiently fine mesh is employed.

In this paper, URANS is used to model the unsteady scalar transport of species or temperature in a turbulent flow duct terminated by an open end. Comparisons of the numerical results for mass fractions and temperature fluctuations due to compositional and thermal perturbations are made with the experimental results. Furthermore, the two perturbation types are contrasted and the underlying assumptions made in their respective numerical methodologies are assessed. No evaluation of sound generation is presented in this work. This study lays the ground for a following investigation on the acoustic response of the scalar non-uniformities for a duct terminated with different nozzle geometries.

The paper is organised as follows. The mechanisms of direct and indirect noise generation are introduced in $\S 2$. The geometric and measurement details relating of the experiments are presented in $\S 3$. The numerical setup, grids, boundary conditions and solvers used for the URANS studies are outlined in $\S 4$. The corresponding results are shown in $\S 5$, where the numerical calculations are compared to experimental measurements. Finally, the results for entropic and compositional perturbations are contrasted in $\S 6$.

\section{Theory for direct and indirect noise generation}

The process of direct and indirect noise generation can be simplified by employing a one-dimensional framework $[24,16]$. The unsteady heat release at the flame location generates direct noise in the form of forward- and backward-propagating acoustic waves $\pi_{d}^{+}$and $\pi_{d}^{-}$, respectively. The combustion process also leads to the generation of entropic waves, $\sigma$, and compositional waves, $\xi$, which are convected downstream towards a region of flow acceleration (such as a nozzle or turbine blades). The unsteady interaction between these convecting waves and a mean-flow gradient is the source of indirect noise, which is comprised of forward- and backward-travelling acoustic waves $\left(\pi_{i}^{+}\right.$and $\left.\pi_{i}^{-}\right)$. Indirect noise is made up of contributions from both the entropic and compositional sources (i.e. $\pi_{i}=\pi_{\sigma}+\pi_{\xi}$ ).

These waves are expressed as fluctuations in the flow variables, which can be decomposed into their mean and fluctuating components (denoted with a macron and 
a prime, respectively: e.g. $\left.p(x, t)=\bar{p}(x)+p^{\prime}(x, t)\right)$. Furthermore, linear fluctuations are considered, such that their amplitude is assumed to be small relative to the mean value (i.e. $p^{\prime} \ll \bar{p}$ ). Assuming constant mean heat capacity, the acoustic, entropic and compositional wave amplitudes are given by:

$$
\pi^{ \pm} \equiv \frac{1}{2}\left(\frac{p^{\prime}}{\bar{\gamma} \bar{p}} \pm \frac{U^{\prime}}{\bar{c}}\right), \quad \sigma \equiv \frac{s^{\prime}}{\bar{c}_{p}}=\frac{p^{\prime}}{\bar{\gamma} \bar{p}}-\frac{\rho^{\prime}}{\bar{\rho}}-\bar{\Psi} Z^{\prime}, \quad \xi \equiv Z^{\prime},
$$

where $p$ is the pressure, $U$ is the flow velocity, $c$ is the sound speed, $s$ is the specific entropy, $c_{p}$ is the specific heat capacity at constant pressure, $\gamma$ is the heat capacity ratio, $\rho$ is the density, $\Psi$ is the chemical potential function, and $Z$ is the mixture fraction [4]. Assuming a compact wave source, the compositional and entropic wave amplitudes for each case can be expressed as:

Gas injection: $\quad \xi=Y_{i}, \quad \sigma=\left(\frac{\bar{s}_{\text {air }}-\bar{s}_{i}}{\bar{c}_{p}}\right) \xi$,

Heat addition: $\quad \xi=0, \quad \sigma \simeq T^{\prime} / \bar{T}$,

where $Y$ is the mass fraction and $T$ is the temperature [24, 36, 37, 38]. Equation (2b) assumes the temperature of the injected gas is equal to the mean flow temperature, $\bar{T}$. The subscript ()$_{i}$ henceforth refers to the properties of the injected gas.

\section{Experimental setup}

The Cambridge Wave Generator (CWG) consists of a modular duct with a mean flow of air which can be perturbed by two sources of inhomogeneities: temperature or composition. The methods for temperature non-uniformity generation and the resulting measurements using fast probes and diagnostics techniques are described in detail in [15] and [17]. Pulsed injection of different gases generate compositional non-uniformities, and the corresponding methods and results are described in [17]. Briefly, in all cases, the mean flow of air was delivered by a mass flow controller connected to a $160 \mathrm{~mm}$ long hose ( $8 \mathrm{~mm}$ ID) into a duct (42.6 $\mathrm{mm}$ ID) of variable length. The description below outlines the experimental setup, while measurement details and uncertainties are available in the original work. A schematic of both setups is shown in Fig. 1.

De Domenico et al. [15] generated temperature spots in a duct of $2.1 \mathrm{~m}$ length using a pulsed electrical heating grid source placed $0.7 \mathrm{~m}$ and $1.4 \mathrm{~m}$ from the inlet and outlet, respectively. For this study, the mass flow rate of air was varied from 


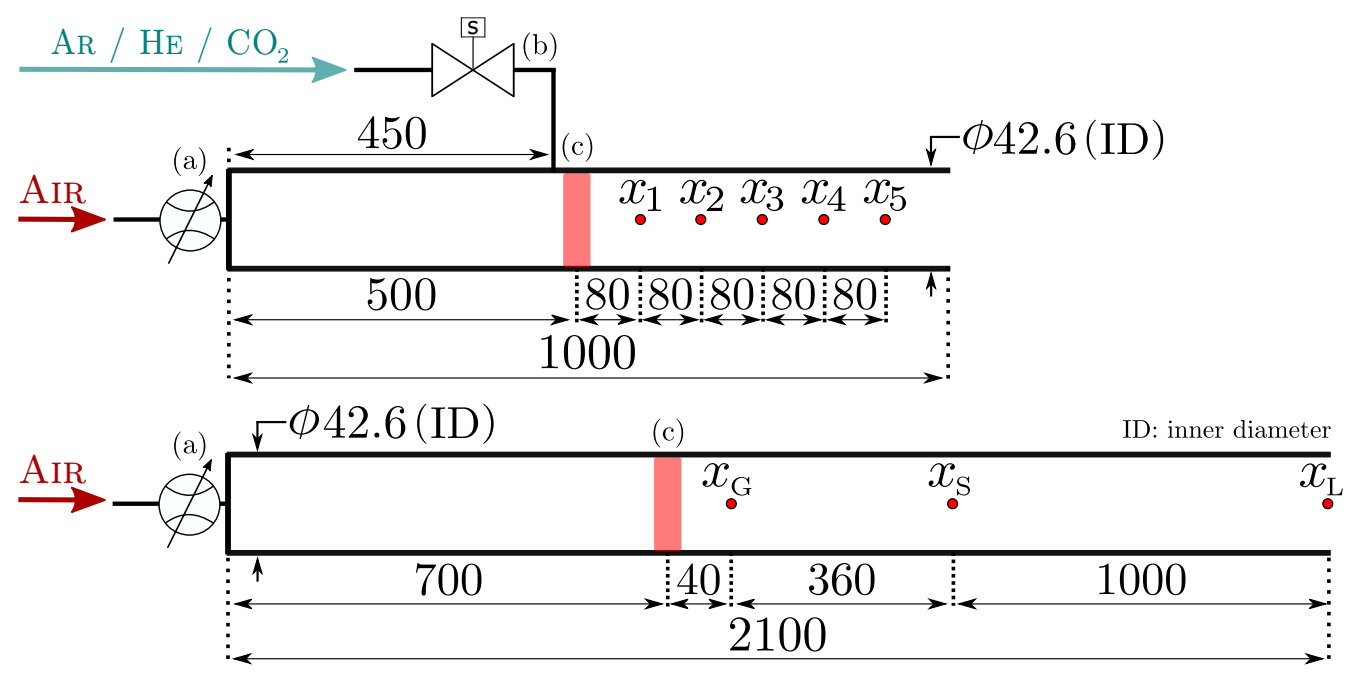

Figure 1: Experimental setup for the LITGS (top) [17] and thermocouple (bottom) [15] measurements with (a) mass flow controller, (b) fast response solenoid valve and (c) heating grid. The probe locations are identified for each experimental campaign $(\bullet)$. All dimensions in $\mathrm{mm}$.

1.54 to $4.93 \mathrm{~g} \mathrm{~s}^{-1}$, providing eight different test cases. Temperature fluctuations were measured at three different locations along the centreline using K-type thermocouples. A hot-film anemometer was used to correct for the long response time of the thermocouples. The probe locations are shown in Fig. 1 (bottom).

More recently, De Domenico et al. [17] generated both temperature and compositional spots using a shorter version of the CWG setup, as shown in Fig. 1 (top). The mean flow of air was fixed at $1.57 \mathrm{~g} \mathrm{~s}^{-1}$. Temperature spots were generated using the same heating grid setup as in [15]. Compositional waves were generated via the radial injection of a secondary gas (argon, carbon dioxide or helium) using an actuated solenoid valve. The tube connecting the injection valve to the main duct was $30 \mathrm{~mm}$ long (4.8 mm ID). Measurements of density, mass fraction and temperature fluctuations due to the passage of the different perturbations were made at five axial locations along the duct centreline using laser-induced grating spectroscopy (LITGS) as shown in Fig. 1 (top).

In both experimental studies, and for both types of perturbation, the pulse frequency was set to $1 \mathrm{~Hz}$, with a pulse duration of $\tau=200 \mathrm{~ms}$. Once generated, the perturbations convected and exited the duct, which was terminated by an open end.

\subsection{Probe volume}

Measurements of the local concentration and temperature are obtained by using LITGS. The technique involves using a gas phase tracer (in this case, biacetyl) which 


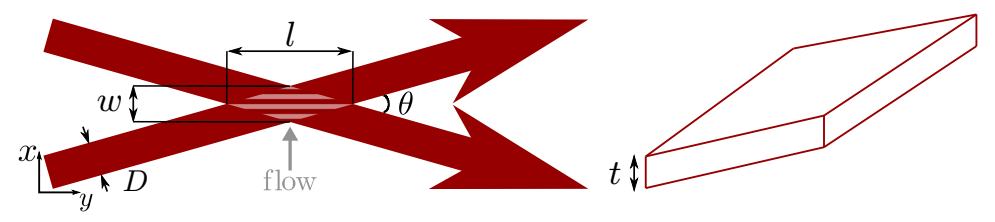

Figure 2: Probe volume schematic.

absorbs the incident light wavelength $(355 \mathrm{~nm})$. Two coherent pulsed beams from the same source are crossed at an angle $\theta$ to generate a grating of wavelength $\Lambda$ over a rhomboic region of length $l$, width $w$, and thickness $t$ as shown in Fig. 2. The laser light is absorbed by the tracer, generating a small perturbation in density, along with corresponding counter-propagating pressure pulses. The density can be determined by a continuous wave (CW) laser with a different wavelength $\lambda_{p}$ incident at an angle $\theta_{p}$ corresponding to the Bragg condition [39]. The effective probed region corresponds to the intersection of the probe volume $V \approx 0.5$ lwt with the probe beam at the Bragg angle.

The outgoing signal from the CW laser beam is captured by a photomultiplier whose frequency is proportional to the local speed of sound. The latter is a function of the mass fraction of the perturbing species and can, therefore, be related to the measured speed of sound. Probe volume dimensions can be estimated via:

$$
l=\frac{2 D \sin \left(\frac{\pi}{2}-\frac{\theta}{2}\right)}{\sin (\theta)}, \quad w=\frac{2 D \cos \left(\frac{\pi}{2}-\frac{\theta}{2}\right)}{\sin (\theta)}
$$

where $D \approx 1 \mathrm{~mm}$ is the beam diameter and the same as the probe volume thickness, $t$. From experiments, $\theta=2.338^{\circ}[17]$. Since $D=t=1 \mathrm{~mm}$, we obtain $l \approx 50 \mathrm{~mm}$ (perpendicular to the flow direction as shown in Fig. 2) and $w \approx 1 \mathrm{~mm}$, for an estimated probe volume region of approximately $25 \mathrm{~mm}^{3}$.

\section{Numerical methodology}

\subsection{Governing equations}

In the modelling of the generation of compositional and entropic waves, besides the mass and momentum equations, two other governing equations are of particular

interest. For the compositional case, we solve the species equation formulated as follows:

$$
\bar{\rho} \frac{D \tilde{Y}}{D t}=\nabla \cdot\left(\left(\frac{\mu_{l}}{S c_{l}}+\frac{\mu_{t}}{S c_{t}}\right) \nabla \tilde{Y}\right)
$$


where $Y$ is the mass fraction, $\mu$ is the viscosity, and $S c$ is the Schmidt number. The material derivative is defined as $\frac{D()}{D t}=\frac{\partial()}{d t}+\mathbf{U} \cdot \nabla()$, where $\mathbf{U}$ is the flow velocity vector. The source term is neglected in the present study since there are no chemical reactions taking place. The subscripts ()$_{l}$ and ()$_{t}$ refer to the laminar and turbulent components, respectively. The Favre- and Reynolds-averaged terms are denoted by a tilde $\left(^{\sim}\right)$ and a macron $\left(^{-}\right)$, respectively.

For the heat addition test case, a volumetric source term, $\dot{S}_{q}$, is incorporated into the total energy equation to represent the power input by Joule heating:

$$
\bar{\rho} \frac{D}{D t}\left(\tilde{e}+\frac{1}{2}|\tilde{\mathbf{U}}|^{2}\right)=-\nabla \cdot\left(p^{*} \tilde{\mathbf{U}}\right)+\nabla \cdot\left(\bar{\rho} \gamma\left(\alpha_{l}+\alpha_{t}\right) \nabla \tilde{e}\right)+\dot{S}_{q},
$$

where $e$ is the internal energy and $\alpha$ is the thermal diffusivity. Lastly, $p^{*}=\bar{p}+\frac{2}{3} \bar{\rho} \tilde{k}$ is a modified mean pressure [40], where $\tilde{k}=\frac{1}{2}|\tilde{\mathbf{U}}|^{2}$ is the specific kinetic energy and $|\tilde{\mathbf{U}}|^{2}=\tilde{\mathbf{U}} \cdot \tilde{\mathbf{U}}$.

\subsection{Solver details}

The open-source CFD software package OpenFOAM 4.1 is used to perform compressible RANS simulations of the steady flow and the unsteady radial gas injection or heat addition in the duct. The solver rhoSimpleFoam is used in both studies to obtain a steady-state solution for the velocity field inside the duct. In all simulations the maximum Courant number is limited to 0.35.

The cases for gas injection pulse and temperature pulse are described separately, since the transient solvers, meshes and operating conditions are different. The transient solvers are both $1^{\text {st }}$ order accurate in time and $2^{\text {nd }}$ order accurate in space, employing the PIMPLE algorithm for the pressure-velocity coupling. More details on the solvers used to obtain the unsteady solutions for the gas injection and heat addition cases are described in $\S 4.3 .1$ and $\S 4.4 .1$, respectively.

\subsection{Gas injection case}

\subsubsection{Numerical setup}

A modified version of the OpenFOAM solver reactingFoam is employed to solve this problem. The standard reactingFoam solver is commonly used in single-phase, multi-species problems; its species transport equation assumes that the ratio between mass diffusivity and momentum diffusivity is unity for both laminar and turbulent terms (i.e. assumes the Schmidt numbers are equal to one, $S c_{l}=S c_{t}=1$ ). The modified version employed here removes this assumption by using Eq. (5) which allows for the implementation of different laminar and turbulent Schmidt numbers, and, therefore, diffusion coefficients depending on the gas simulated, as shown in 
Table 1. The laminar values are based on the assumption of binary diffusion coefficients and computed with respect to air according to the Chapman-Enskog theory [41]. Turbulent numbers are assumed to be 0.7 as is done in commercial software and to ensure unity turbulent Lewis number [42]. Buoyancy effects are also accounted

for via the incorporation of the respective gravitational terms in the momentum and energy equations. Thermal (Soret) diffusion is not included.

The present work deals with steady and unsteady simulations involving a variety of Mach and Reynolds numbers. The main flow of air has a fixed flow rate of $\overline{\dot{m}}=1.57 \mathrm{~g} \mathrm{~s}^{-1}\left(\bar{U}=0.92 \mathrm{~m} \mathrm{~s}^{-1}, \bar{M}=0.003\right)$ falling under the transitional regime $(R e=2580)$, while the secondary gases produce cross-flow jets with Reynolds numbers ranging from 790 to 8350 and Mach numbers ranging from 0.02 to 0.07 (for helium and argon, respectively) as shown in Table 1 . The $k-\omega$ SST model [43] with wall functions is used to model the turbulent fluctuations in the flow. Tkatchenko et al. [33] showed that the SST turbulence model was the most accurate model for simulating this type of problem using URANS, based on comparisons with LIF and LDA measurements. Since the temperature in the domain is constant throughout the simulation, the dynamic viscosity, $\mu$, and the Prandtl number, $P r$, are also constant and specified for each gas as inputs; these are outlined in Table 1. The dynamic viscosity and Prandtl number for air are set to $\mu=18.3 \mu \mathrm{Pa}$ s and $\operatorname{Pr}=0.7$, respectively.

Each simulation was run using 96 cores and required a maximum of 4.5 hours of wall clock time to obtain statistics for $1 \mathrm{~s}$ of physical time.

\subsubsection{Modelling of injection}

The solenoid valve delivers a very fast pulse $(200 \mathrm{~ms}$, with a response time of around $10 \mathrm{~ms}$ ). Given that direct measurements of the instantaneous mass flow rate are challenging, other methods are required to estimate both the total and instantaneous perturbation. In this study, the phase-averaged mass fraction measurements obtained via LITGS due to the passage of 30 injections is used to drive the numerical simulations. Specifically, the injected mass flow rates are evaluated assuming full radial mixing at the most upstream location, $x_{1}$. In that case, species conservation for injected species $i$ yields:

$$
\dot{m}_{i}^{\prime}=\frac{Y_{i_{\max }}\left(x_{1}\right)}{1-Y_{i_{\max }}\left(x_{1}\right)} \dot{\bar{m}}
$$

where $Y_{i_{\max }}$ is the maximum mass fraction measured in time at $x_{1}$. The estimated injected flow rates for each gas are shown in Table 1. 


\begin{tabular}{ccccccccccc} 
Gas & $\dot{m}_{i}^{\prime}$ & $R e_{i}$ & $M_{i}$ & $J_{i}$ & $M W$ & $\rho_{i} / \bar{\rho}$ & $\Delta s / \bar{c}_{p}$ & $\mu$ & $S c_{l}$ & $S c_{t}$ \\
& {$\left[\mathrm{~g} \mathrm{~s}^{-1}\right]$} & {$[-]$} & {$[-]$} & {$[-]$} & {$\left[\mathrm{g} \mathrm{mol}^{-1}\right]$} & {$[-]$} & {$[-]$} & {$[\mu \mathrm{Pa} \mathrm{s}]$} & {$[-]$} & {$[-]$} \\
\hline $\mathrm{Ar}$ & 0.692 & 8350 & 0.07 & 864 & 39.948 & 1.69 & 2.98 & 22.3 & 0.812 & 0.7 \\
$\mathrm{He}$ & 0.058 & 790 & 0.02 & 60 & 4.003 & 0.17 & -24.33 & 19.6 & 0.220 & 0.7 \\
\hline
\end{tabular}

Table 1: Estimated injected mass flow rate $\left(\dot{m}_{i}^{\prime}\right)$, Reynolds number $\left(R e_{i}\right)$, Mach number $\left(M_{i}\right)$, jet-to-crossflow momentum flux ratio $\left(J_{i}\right)$, molecular weight $(M W)$, density ratio $\left(\rho_{i} / \bar{\rho}\right)$, entropiccompositional coupling term in Eq. (2b) $\left(\Delta s / \bar{c}_{p}\right)$, dynamic viscosity $(\mu)$, and laminar and turbulent Schmidt numbers $\left(S c_{l}, S c_{t}\right)$ for each gas at $T=293 \mathrm{~K}$ [44]. The mean flow rate of air is $\overline{\dot{m}}=1.57 \mathrm{~g} \mathrm{~s}^{-1}\left(\bar{U}=0.92 \mathrm{~m} \mathrm{~s}^{-1}, R e=2580\right)$ for all cases. For air, $\mu=18.3 \mu \mathrm{Pa} \mathrm{s}$ and $\operatorname{Pr}=0.7$.

\subsubsection{Grid and geometry}

For the gas injection cases, the full domain is discretised using a structured multiblock mesh, employing 1.6 million cells as shown in Fig. 3 (top). Symmetry boundary conditions are applied at the jet centre plane allowing for reduced computational costs. The structures generated by a jet in crossflow are three-dimensional [30], therefore the planar symmetry condition employed is an approximation. Since the focus of this work is the convection and dispersion of the compositional wave once it has been injected, the reduced accuracy of the injection process is accepted.

Before applying the unsteady injection, a test case is run with the full geometry for the steady undisturbed flow with the fixed inlet mass flow rate for all injection cases $\left(\overline{\dot{m}}=1.57 \mathrm{~g} \mathrm{~s}^{-1}\right)$. The velocity profile $0.1 \mathrm{~m}$ upstream of the injection location is observed to be fully developed, therefore, a reduced geometry is used starting at the stated location, where the inlet condition imposed for velocity corresponds

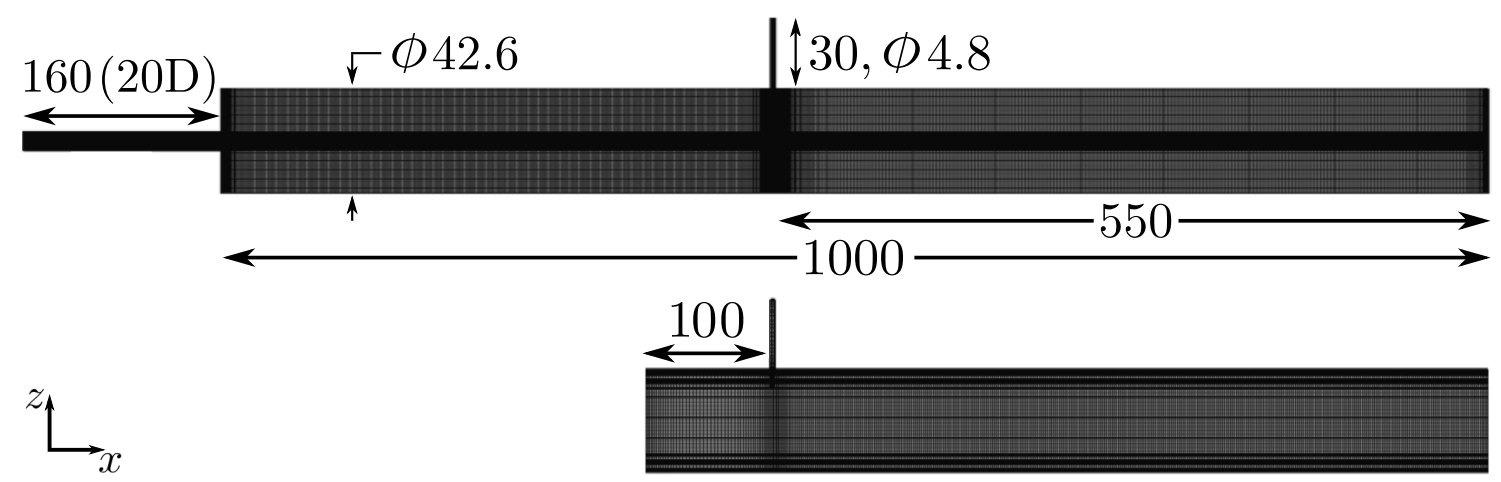

Figure 3: Meshes for the full (top) and reduced (bottom) configurations used in the gas injection cases on the $x-z$ mid-plane. Figures scaled axially by a factor of 0.33 . Dimensions in $\mathrm{mm}$. 
to the velocity profile measured in the original steady-state calculation. Figure 3

\subsubsection{Modelling of source term, $\dot{S}_{q}$}

The source term, $\dot{S}_{q}$, in Eq. (6) represents the heat transfer per unit volume arising from the heating grid. This is determined by estimating the total effective power delivered during the unsteady heating interval based on temperature measurements. This is analogous to the block deposition model referenced in $\S 1$, in which all the energy is assumed to be transferred into a single region [20, 23, 24, 25, 26]. The heat convected into the air, $\dot{Q}_{c}$, and volumetric source term, $\dot{S}_{q}$, can be described by a semi-empirical formulation derived from fitting to the average of an ensemble of 
experimental measurements:

$$
\begin{gathered}
\dot{Q}_{c}= \begin{cases}a^{2} b \dot{Q}_{0}\left(1-e^{-\frac{t}{\tau}}\right), & \text { for } t \leq t_{t} \\
b \dot{Q}_{0}\left(1-e^{-\frac{t}{\tau}}\right), & \text { for } t_{t}<t \leq t_{p} \\
h A_{w}\left(\left(T_{w_{\max }}-\bar{T}\right) e^{-\frac{\left(t-t_{p}\right)}{\tau}}\right), & \text { for } t>t_{p}\end{cases} \\
\qquad \dot{S}_{q}=\frac{\dot{Q}_{c}}{V},
\end{gathered}
$$

where $\dot{Q}_{0}$ is the steady-state power input, $T_{w}$ is the wire temperature (assumed to be uniform), $\bar{T}$ is the mean flow temperature (assumed to be $293 \mathrm{~K}$ ), $h$ is the convective heat transfer coefficient for flow over a cylinder [47] (calculated from the Nusselt number and gas properties using Hilpert correlations [48]), $A_{w}$ is the cross sectional area of each wire (computed from the wire thickness, assumed to be $56 \mu \mathrm{m}$ ), $\tau$ is the time constant (determined by $\tau=\rho_{w} d_{w} c_{w} / 4 h$, assuming the properties are independent of temperature), $t_{p}$ is the unsteady heating duration (set to $200 \mathrm{~ms}$ ) and $t_{t}$ is the transient current surge duration (measured experimentally to be $0.015 \mathrm{~s}$ ). This is shown in Fig. 4 (a). The additional power supplied to the air during the transient period, $t_{t}$, can be modelled by a transient multiplier factor, $a$, for the steady state current, $\bar{i}$. This factor appears in Fig. 4 (b) as $a^{2}$ since $\dot{Q} \propto i^{2}$ and was optimised to match the direct noise produced in the experiments (not presented in this paper). For all computations, a was set to 1.50. A bulk multiplier factor for the total power, $b$, was then included to account for the loss of overall power to the copper metal holder during the pulse duration. Mühlbauer et al. [19] presented a similar study on the effect of the energy supplied to the overall temperature and

(a)

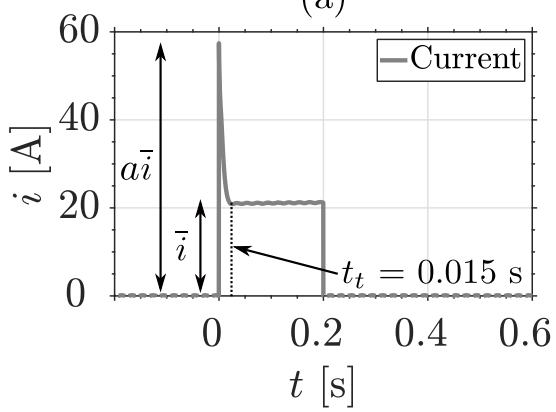

(b)

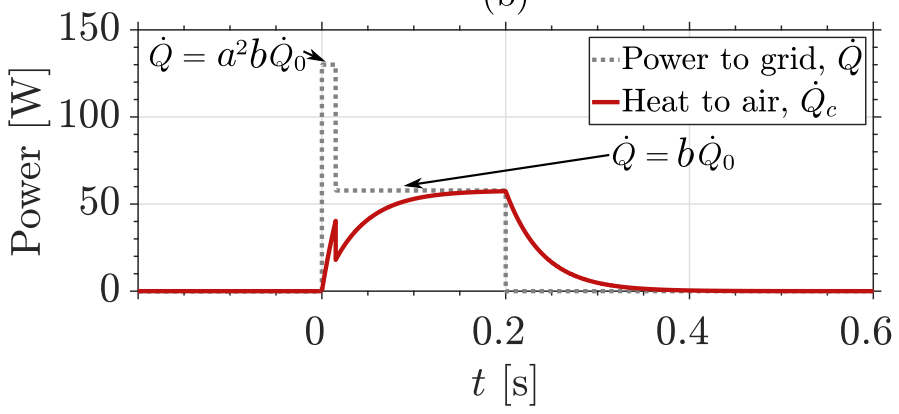

Figure 4: (a) Current delivered to grid driving system (-); (b) Modelled power input (- - ) and convected power $(-)$ for case $8\left(\bar{U}=2.56 \mathrm{~m} \mathrm{~s}^{-1}\right)$. Heat addition from $t=0$ to $0.2 \mathrm{~s}$. 


\begin{tabular}{cccccc} 
Case & $\bar{U}$ & $\begin{array}{c}R e \\
{[-]}\end{array}$ & $\begin{array}{c}b \\
{[-]}\end{array}$ & $\begin{array}{c}\dot{Q}_{c_{\max }} \\
{[\mathrm{W}]}\end{array}$ & $\begin{array}{c}\tau \\
{[\mathrm{s}]}\end{array}$ \\
\hline 1 & 0.88 & 2510 & 0.1250 & 52.94 & 0.0619 \\
2 & 1.12 & 3220 & 0.1259 & 53.83 & 0.0572 \\
3 & 1.24 & 3580 & 0.1263 & 54.24 & 0.0559 \\
4 & 1.49 & 4350 & 0.1272 & 54.99 & 0.0509 \\
5 & 1.75 & 5160 & 0.1281 & 55.66 & 0.0477 \\
6 & 1.98 & 5960 & 0.1290 & 56.19 & 0.0520 \\
7 & 2.27 & 6930 & 0.1300 & 56.81 & 0.0426 \\
8 & 2.56 & 8060 & 0.1310 & 57.38 & 0.0403
\end{tabular}

Table 2: Summary of numerical test cases: bulk flow velocity $(\bar{U})$, Reynolds number $(R e)$, bulk power factor $(b)$, maximum heat transferred to air $\left(\dot{Q}_{c_{\max }}\right)$ and time constant $(\tau)$. Transient factor $a=1.50$ for all cases.

pressure fluctuations measured. Table 2 summarises the values for each test case.

The volume of the modelled computational heating grid, $V$, is approximated as the product of the duct area and the heating module length (assumed to be $10 \mathrm{~mm}$ ). Experimental temperature measurements at probe location $x_{G}(0.04 \mathrm{~m}$ downstream of the heating grid) were used to validate the modelling coefficients for each bulk flow velocity. Eight test cases are selected to study the effect of the mean flow rate, and, therefore, turbulence, on the convection of the entropic waves.

\subsubsection{Grid and geometry}

The geometry modelled in the simulations is shown in Fig. 5. In this study, we were also interested in studying the acoustic response of the system (not presented here) so the geometry was not reduced as per $\S 4.3 .3$. The full grid consists of a rotationally symmetric grid represented by a segment of $2.5^{\circ}$ for a duct of $42.6 \mathrm{~mm}$ ID and $2.1 \mathrm{~m}$ length. This simplification was also employed by Mühlbauer et al. [20] and Leyko et al. [23], albeit using a $10^{\circ}$ slice as it did not show a decrease in the accuracy of the results obtained from the simulations. The mesh shown in Fig. 5 has 26,350 cells and is divided into three blocks (pre-heating grid, heating grid, and postheating grid), each with varying numbers of cells in the axial direction (120, 7 and 400 cells, respectively). There are 50 cells in the radial direction (with a $50 \%$ expansion ratio) and one cell in the azimuthal plane (due to the axisymmetry assumption). The mesh was refined near the heating grid to capture the steep temperature gradients. A mesh sensitivity study was undertaken to ensure that the wave characteristic times and amplitudes were independent of the mesh. 


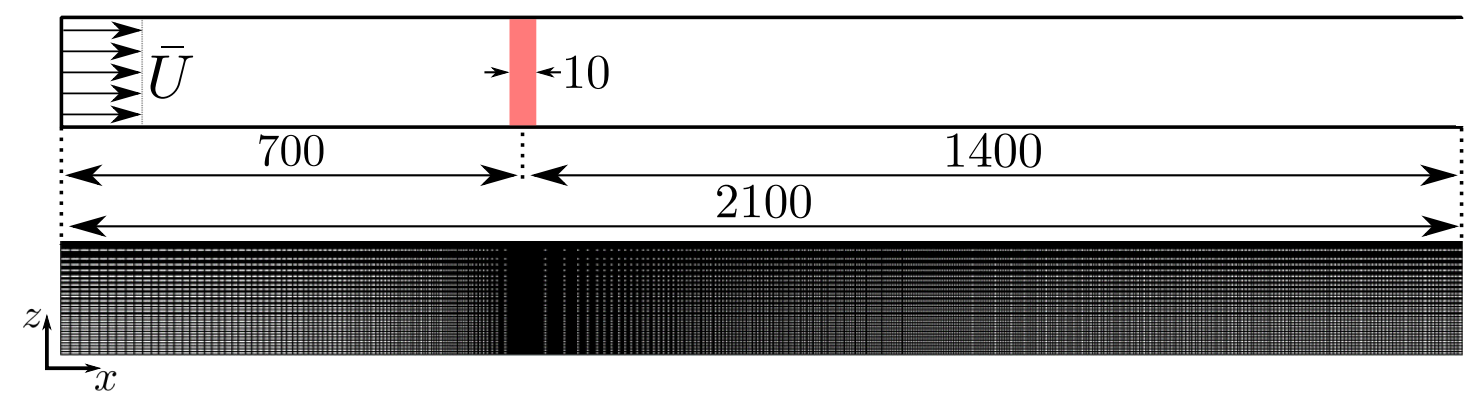

Figure 5: Numerical setup for the heat addition case on the $x-z$ mid-plane: schematic (top) and mesh (bottom). Heating grid modelled as volume with length of $10 \mathrm{~mm}$. Dimensions in $\mathrm{mm}$.

\subsubsection{Boundary conditions}

A uniform velocity profile is used for the inlet flow and zero pressure gradient and no-slip velocity boundary conditions are imposed at the wall. A zero gradient pressure condition is imposed at the inlet and a mean atmospheric pressure is enforced at the outlet. The wall is assumed to be isothermal $\left(T_{W}=293 \mathrm{~K}\right)$.

\section{$1 D$ radial conduction $\mathcal{E}$ convection boundary condition.}

To assess the influence of heat loss on the convection of an entropic wave, a onedimensional linearised conduction and convection boundary condition is derived. The temperature boundary condition is determined by assuming radial conduction across the thin duct thickness, whilst axial conduction is neglected [47]:

$$
q_{r}^{\prime}=\Theta\left(T_{W}(x)-T_{\infty}\right), \quad \Theta=\left(\frac{\ln \left(r_{2} / r_{1}\right)}{2 \pi \lambda_{W}}+\frac{1}{h_{\infty} 2 \pi r_{2}}\right)^{-1},
$$

where $T_{W}(x)$ is the inner wall temperature, $T_{\infty}$ is the ambient temperature, $\lambda_{W}$ is the metal wall conductivity, and $r_{1}$ and $r_{2}$ are the inner and outer radii.

Although the CWG rig is made of both PVC and steel modules, the thermal conductivity of the wall is assumed to be $\lambda_{W}=50.2 \mathrm{~W} \mathrm{~m}^{-1} \mathrm{~K}^{-1}$ (steel). The ducts are not perfectly round, but a mean thickness of $3.2 \mathrm{~mm}$ is assumed.

It is also important to consider the possible range of values for the free convection heat transfer coefficient, $h_{\infty}$. The convection heat transfer coefficient for the free convection of gases typically ranges from 2 to $25 \mathrm{~W} \mathrm{~m}^{-2} \mathrm{~K}^{-1}$ according to Bergman et al. [47]; values within this range are investigated and presented in §5.2.3. The change in thermal conductivity is found to be negligible due to the small range of temperatures investigated (293-320 K). 


\section{Results}

\subsection{Gas injection}

From the three cases studied by De Domenico et al. [15], the injections of argon and helium have been selected for this numerical work as they are both inert gases with one being heavier and the other lighter than air.

\subsubsection{Compositional source field evolution}

The injection and convection of the compositional waves is shown at different time steps in Fig. 6. Two orthogonal mid-planes are presented with the corresponding flow-field distributions coloured by the entropic and compositional wave amplitude expressions given in Eqs. $(2 a-b)$. The injection occurs from $t=0$ to $200 \mathrm{~ms}$.

Figure 6 shows that the mass fraction of argon added during the injection process reaches a maximum of $50 \%(\xi=0.5)$. Comparatively, helium produces a much weaker compositional disturbance $(\xi=0.1)$ due to the low molecular mass of helium (one order of magnitude lower than air) and the reduced injected mass flow rate, which is only $4 \%$ of the mean air flow. However, it still generates a significant entropic perturbation $(\sigma=-2.4)$ owing to the considerable specific entropy difference between air and helium $\left(\Delta s / \bar{c}_{p}=-24.33\right)$. In contrast, argon has a lower specific entropy difference to air $\left(\Delta s / \bar{c}_{p}=2.98\right)$ and, therefore, needs a larger injected mass flow rate to produce the same absolute entropic disturbance as helium. For the test cases presented, and despite the larger injected flow rate, argon produces a weaker absolute entropic disturbance $(\sigma=1.5)$ than helium.

As the jets cross the duct, they are both diverted by the axial momentum of the mean flow according to their respective jet-to-crossflow momentum flux ratios, $J_{i}$. The argon jet has the largest momentum flux ratio $\left(J_{\mathrm{Ar}}=864\right)$, impinging on the opposing side of the duct and producing a toroidal vortex upstream of the injection location (see $t=25-100 \mathrm{~ms}$ on the $x-z$ and $x-y$ mid-planes). In the case of helium, however, the momentum flux ratio is one order of magnitude smaller $\left(J_{\mathrm{He}}=60\right)$ and the jet is instead promptly advected by the surrounding air flow. The jet barely touches the opposing side of the duct, producing a minimal vortex upstream of the injection location (see $t=100 \mathrm{~ms}$ on the $x-z$ mid-plane). By $t=250 \mathrm{~ms}$, radial mixing at probe location $x_{1}$ is completed in the core of the argon plume, but not in the core of the helium plume; this is more clearly seen on the $x-y$ mid-plane.

The evolution in time of the centreline entropic and compositional perturbations downstream of the injection location is shown in Fig. 7 for both gases. The transport of the inhomogeneities is controlled by advection, given by the mean slope of the $t-x$ plot along an iso-concentration line which coincides with the velocity of the centreline flow $\left(U_{c l} \approx 1.1 \mathrm{~m} \mathrm{~s}^{-1}\right)$. In fact, buoyancy and molecular diffusion are both 

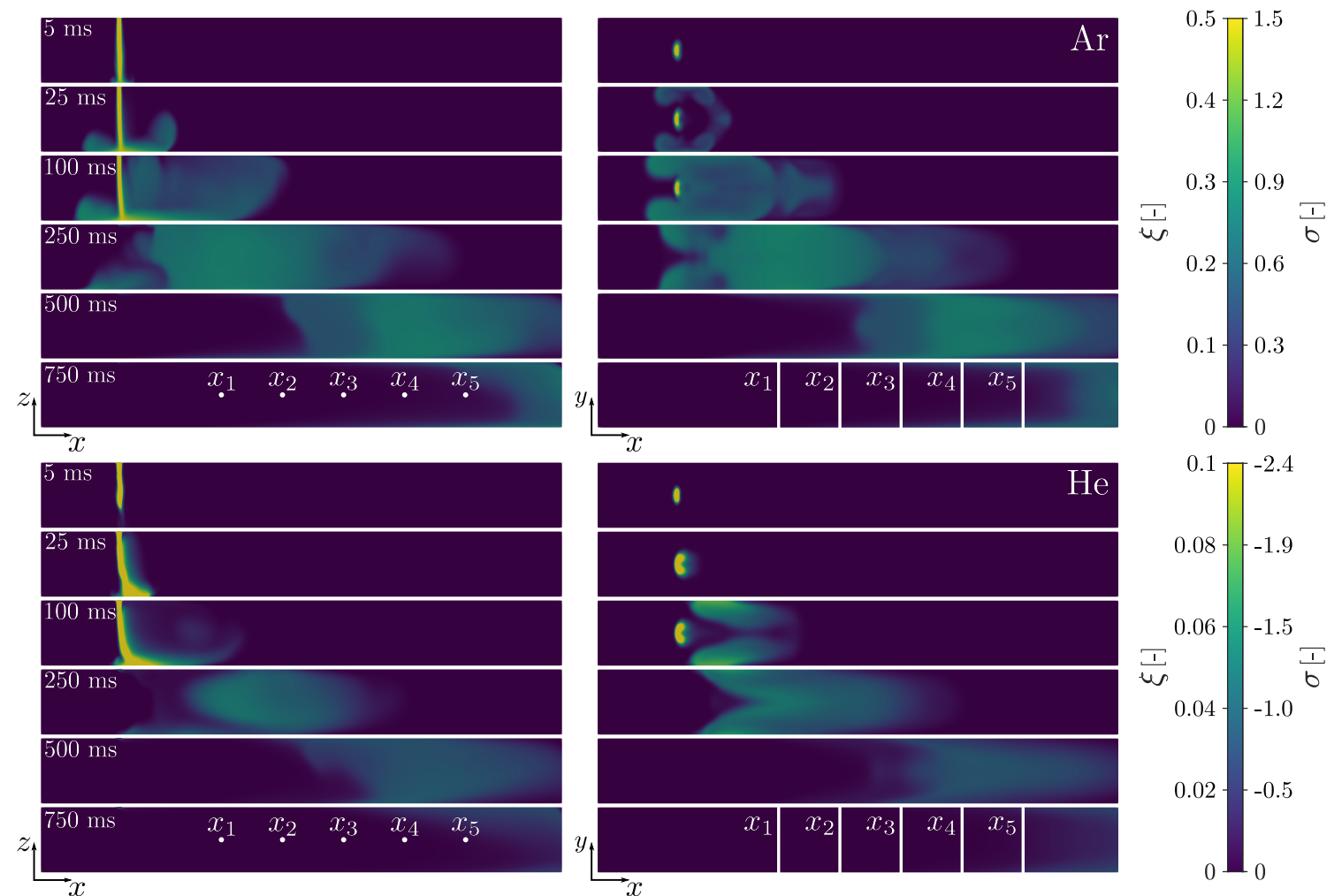

Figure 6: Evolution of the compositional and entropic wave amplitude distribution for argon (top) and helium (bottom) on the $x-z$ mid-plane (left) and $x-y$ mid-plane (right). Probe volume schematics are shown to scale (based on Figs. 1 and 2): $x_{1}=0.13 \mathrm{~m}, x_{2}=0.21 \mathrm{~m}, x_{3}=0.29 \mathrm{~m}$, $x_{4}=0.37 \mathrm{~m}$ and $x_{5}=0.45 \mathrm{~m}$ downstream of the injection location. Figures scaled axially by a factor of 0.5. Injection from $t=0$ to $200 \mathrm{~ms}$.

negligible since accounting for these effects showed minimal differences in the resulting mass fraction signatures (not shown here). All plumes are, therefore, convected downstream at similar rates. However, there are evident dissimilarities between the two cases. In particular, the results for the injection of helium show a highly localised region of maximum amplitude (0.10-0.15 m downstream of the injection location). In the case of argon, on the other hand, the region of maximum amplitude is convected at the centreline from the injection point to the outlet (see $t=100-250 \mathrm{~ms}$ on the $x-y$ mid-plane). This difference is associated with the contrasting jet momenta and the resulting radial mixing dissimilitude discussed previously. Finally, and as an additional consequence of the larger injection flow rate, the injection of argon produces a longer disturbance plume than the injection of helium. Most notably at $t=0.3 \mathrm{~s}$, the maximum disturbance length is $0.30 \mathrm{~m}$ for the helium case and $0.45 \mathrm{~m}$ 


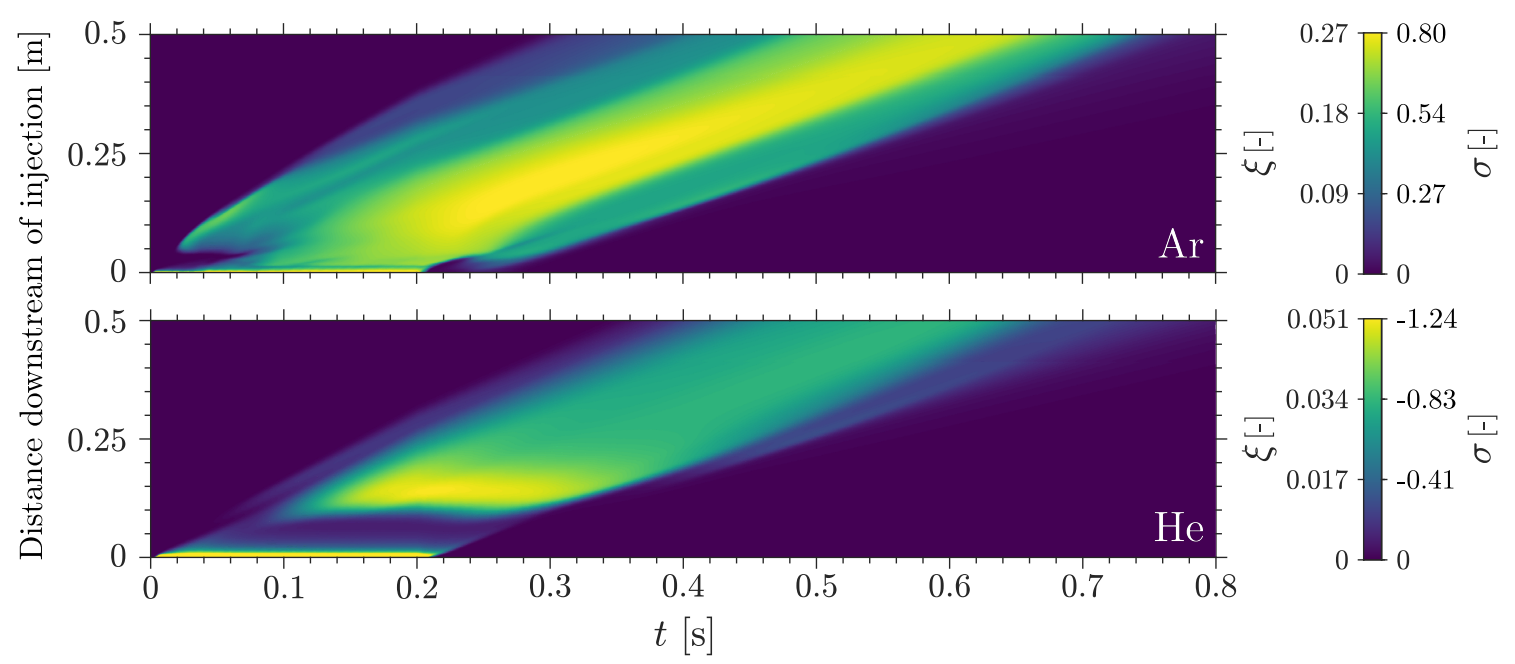

Figure 7: Evolution of the centreline compositional and entropic perturbations downstream of the injection location for argon (top) and helium (bottom). Injection from $t=0$ to $0.2 \mathrm{~s}$.

for the argon case (30\% and $45 \%$ of the duct's total length, respectively).

\subsubsection{Comparison with experimental measurements}

The numerical results for the mass fractions of the injected gases were sampled at a frequency of $1 \mathrm{kHz}$ at selected axial positions downstream of the injection point shown in Fig. 1. The LITGS results are averaged across a probe volume [17]. In the comparison of the simulation and experimental measurements shown in Fig. 8, three sampling methodologies are studied: centreline point, probe volume-averaged (described in §3.1) and cross-sectional area-averaged $\left(\int_{S} Y_{i} d s / \int_{S} d s\right)$.

In all cases, the advection of the plume is well captured by the numerical models. The width of the axially dispersing wave is similar, however, the numerical results become less symmetric downstream of the injection region, whereas the experimental results show a fairly invariable profile. Differences in characteristic convection times appear for both gases further downstream, but more so for helium. Specifically, the numerical results lag the experimental measurements by $0.05-0.10 \mathrm{~s}$. The mismatch in the arrival time of the wave at the probe and in the time at which the peak is measured would suggest a lower predicted velocity at the centreline relative to the experiments. However, no velocity measurements are currently available for comparison.

Numerical results for argon show good agreement with experiments at $x_{1}$ for all three sampling methodologies, specifically in capturing the leading and trailing edge signatures, as well as the wave's convective time and peak perturbation amplitude. 


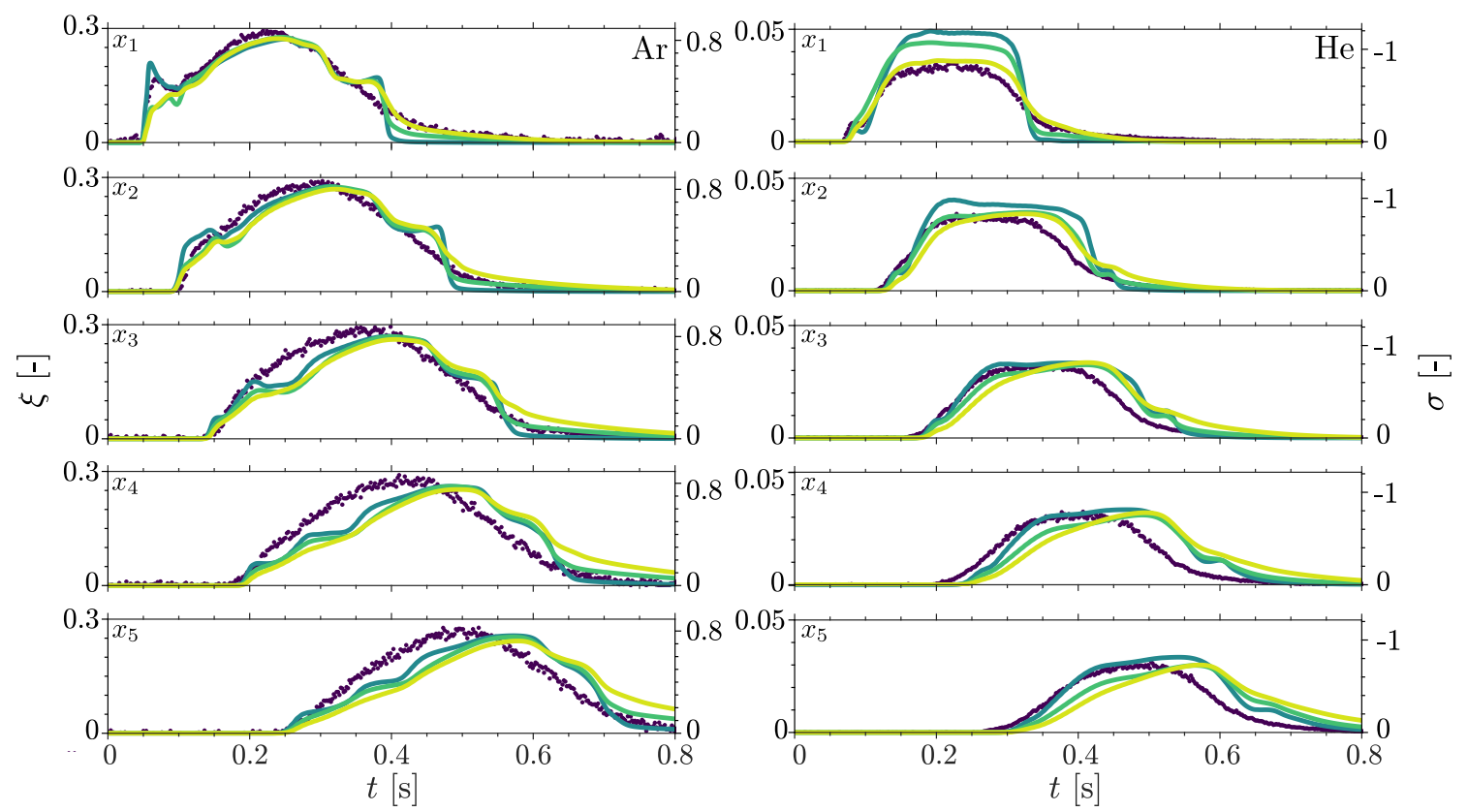

Figure 8: Time series of the centreline compositional and entropic perturbations for argon (left) and helium (right) at $x_{1}=0.13 \mathrm{~m}, x_{2}=0.21 \mathrm{~m}, x_{3}=0.29 \mathrm{~m}, x_{4}=0.37 \mathrm{~m}$ and $x_{5}=0.45 \mathrm{~m}$ downstream of the injection location. Comparison between experimental data using LITGS phaseaveraged from 30 shots $(\bullet)[17]$ and the numerical results using URANS: centreline point $(-)$, probe volume average $(-)$ and cross-sectional area average $(-)$.

The leading edge peak associated with the initial vortex at injection is well predicted by the point measurement but seems to be been smeared out by the probe volume and cross-sectional area averages. Numerical results capture a trailing edge structure which isn't present in the experimental data; this is more clearly visible in probes $x_{1}$ and $x_{2}$. At $x_{2}$, the probe volume-averaged result follows the experimental data more closely, with the point measurement slightly overpredicting the leading edge profile. At the three remaining downstream probe locations, however, all sampling methodologies incur a slight discrepancy in the time at which the peak value is measured. Despite this, the numerical results at $x_{3}, x_{4}$ and $x_{5}$ agree with experiments in terms of the wave's arrival time and peak perturbation amplitudes.

For helium, the width of the plume and peak perturbation amplitude are well captured. The maximum perturbation amplitude at $x_{1}$ agrees with the cross-sectional area-averaged simulation results, whereas it is overpredicted by both the point and probe volume-averaged results. The mass fraction profiles for helium in Fig. 6 show that the wave is not fully mixed across the duct's diameter at $x_{1}(t=100-250 \mathrm{~ms})$; it 
only becomes radially homogeneous further downstream $(t=500 \mathrm{~ms})$, as previously discussed in §5.1.1. This is in contrast to the experimental data which shows the same amplitude being convected through all probe locations (see Figs. 8 and 12). Since the peak amplitudes agree at the probes further downstream for helium $\left(x_{2}\right.$ to $\left.x_{5}\right)$, as well as at all probe locations for argon $\left(x_{1}\right.$ to $\left.x_{5}\right)$, the error at $x_{1}$ for helium is not due to the mass flow rate estimation. Instead, it is due to the planar symmetry assumption which, for this particular case, affects the mixing and leads to the flow requiring a longer convective length to fully homogenise. This isn't an issue with argon since the high jet momenta generates significant mixing such that the mass fraction rapidly becomes homogeneous before reaching $x_{1}$. Therefore, the simulated compositional amplitude for argon does not vary when convected by the mean flow (see Figs. 7, 8 and 12).

\subsection{Heat addition}

\subsubsection{Entropic source field evolution}

The generation and convection of the entropic waves is shown at different time steps for case $1\left(\bar{U}=0.88 \mathrm{~m} \mathrm{~s}^{-1}\right)$ and case $8\left(\bar{U}=2.56 \mathrm{~m} \mathrm{~s}^{-1}\right)$ in Fig. 9. The flow-field distribution on the $x-z$ mid-plane is coloured by the entropic wave amplitude expression given in Eq. $(3 b)$ (since $\xi=0$ ). The heat addition occurs from $t=0$ to $200 \mathrm{~ms}$

The inlet velocity to the heater consists of a fully developed velocity profile. As a result, velocities and mass fluxes are lower in the boundary layer; therefore, a uniform heat flux results in the maximum temperature fluctuation occurring near the wall.
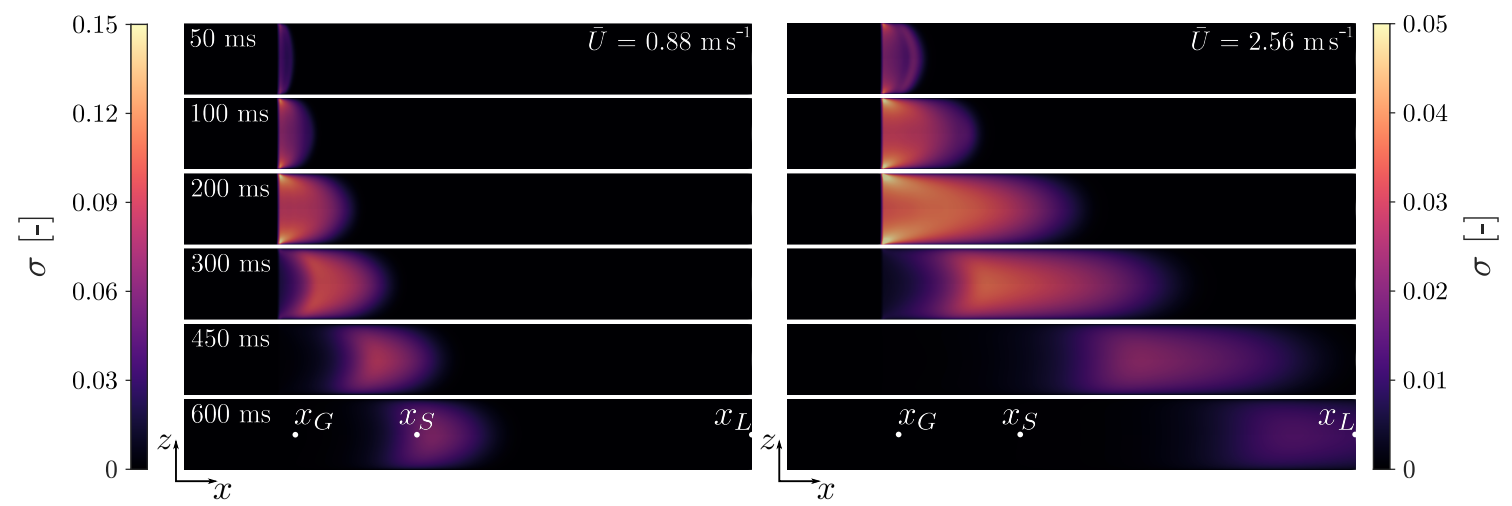

Figure 9: Evolution of the entropic wave amplitude distribution on the $x-z$ mid-plane for case 1 (left) and case 8 (right) $(\xi=0)$. Part of the upstream section has been omitted; $1.68 \mathrm{~m}$ of the duct is shown. Probe locations shown to scale: $x_{G}=0.04 \mathrm{~m}, x_{S}=0.36 \mathrm{~m}$, and $x_{L}=1.4 \mathrm{~m}$ downstream of the heating grid. Figures scaled axially by a factor of 0.4 . Heat addition from $t=0$ to $200 \mathrm{~ms}$. 


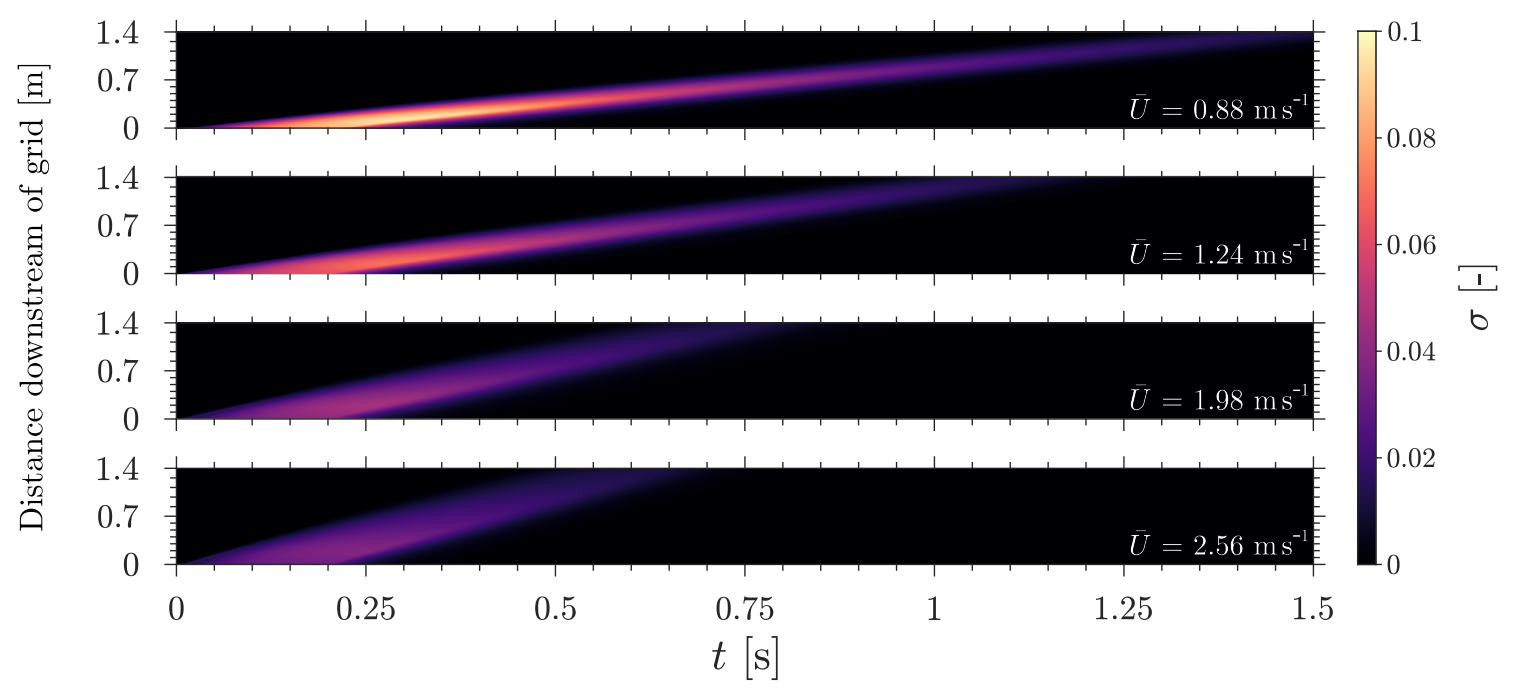

Figure 10: Evolution of the centreline entropic perturbations downstream of the heating grid for cases $1,3,6$ and $8(\xi=0)$. Heat addition from $t=0$ to $0.2 \mathrm{~s}$.

Downstream of the heating grid, the temperature in the boundary layer proceeds to dissipate faster than at the centre of the pipe due to heat transfer to the wall. The wave disperses axially in time, owing to the axial velocity profile, thermal diffusion and heat loss to the wall: starting from the initial $10 \mathrm{~mm}$ heating module length, the perturbations become significantly longer during the period of heat addition. Looking specifically at $t=450 \mathrm{~ms}$ (at which point no more heat is being transferred to the mean flow of air), the maximum disturbance length is $310 \mathrm{~mm}$ for case 1 and $730 \mathrm{~mm}$ for case 8 (15\% and $35 \%$ of the duct's total length, respectively). For these operating conditions and this convective length $(1.4 \mathrm{~m})$, the remaining perturbation at the outlet is very small $(\sigma \approx 0.01)$.

The evolution of the centreline entropic perturbations downstream of the grid is shown in Fig. 10. The behaviour is similar to that of the compositional plume. The entropy spot moves along the duct according to the centreline velocity and, therefore, higher bulk velocities lead to higher slopes on the $t-x$ plane. The width of the entropic spot at the centreline increases as discussed above owing to axial dispersion. Furthermore, higher bulk velocities lower the peak temperature for the given power delivered, as outlined in Table 2; this trend is also evident in Fig. 11. Finally, and as discussed for Fig. 9, the energy is dissipated towards the end of the duct owing to heat transfer to the wall. 


\subsubsection{Comparison with experimental measurements}

The comparison between URANS and thermocouple results for four test cases is shown in Fig. 11 (a). The peak entropic perturbation at $x_{S}$ is matched for all cases; this is expected since the power profile was scaled to agree with near-grid measurements. The comparison further downstream shows differences in the decay of the peak temperature with time: at $x_{S}$, the simulations slightly over-predict the peak measurements, while at $x_{L}$ they under-predict. Despite the mismatch in peak amplitudes, the overall signature of the wave at the centreline is best captured at $x_{S}$ and $x_{L}$, whereas at $x_{G}$ the trailing edge of the heat pulse decays much faster than the thermocouple measurement.

Figure 11 (b) compares the URANS results to both thermocouple and LITGS measurements [17] at two positions. Since $x_{5}-x_{S}$ are at the same distance downstream from the heating grid and $x_{1}-x_{G}$ are separated only by $0.04 \mathrm{~m}$, this figure provides a fair comparison in terms of perturbation amplitude and signature. At the first position, the peak entropic perturbation using LITGS is slightly lower than the URANS and thermocouple results. At the second position, however, the three results are in accordance. Although the agreement is good overall, it is clear to see that the experimental techniques can yield slightly different temperature profiles for the same heat input.

465 The shapes and arrival times of the temperature perturbations shown in Fig. 11 (a) are in better agreement at higher bulk flow velocities. Similar to what is seen in the gas injection cases, the discrepancy in timings would suggest a lower predicted velocity at the centreline relative to the experiments. Likewise, no velocity measurements are currently available for comparison. Additionally, the Reynolds numbers in the duct range from 2500 to 8100 , a relatively transitional region within which the URANS two-equation turbulence model may not fully capture the dispersion effects. In fact, the temperature results at $x_{S}$ were found to be quite sensitive to turbulence models (not shown here). Furthermore, there could also be differences due to the wall temperature boundary condition. Specifically, a constant temperature (isothermal condition) may not fully represent the behaviour at the wall.

\subsubsection{Heat loss study}

To investigate the effect of the temperature condition at the wall on the results, a sensitivity study to the boundary condition derived in $\$ 4.4 .4$ is presented. Figure 11 (c) shows the effect of altering the boundary conditions from a fixed wall temperature of $293 \mathrm{~K}$ (isothermal) to the heat transfer formulation using different convection coefficients $\left(2\right.$ and $\left.25 \mathrm{~W} \mathrm{~m}^{-2} \mathrm{~K}^{-1}\right)$. Results presented are for case 1 $\left(\bar{U}=0.88 \mathrm{~m} \mathrm{~s}^{-1}\right)$. 
(a)
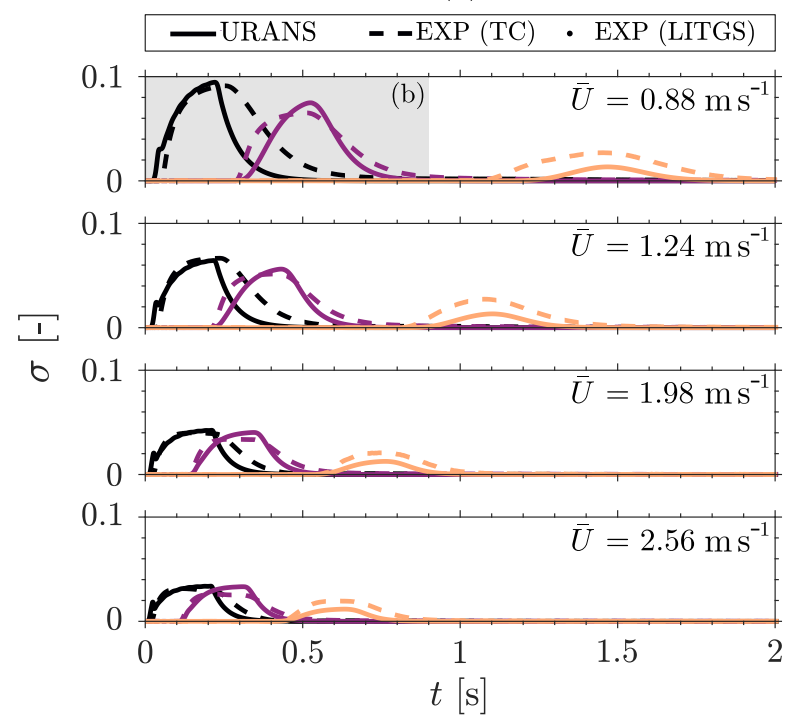

(b)

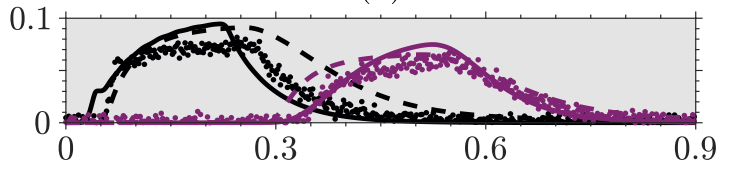

(c)
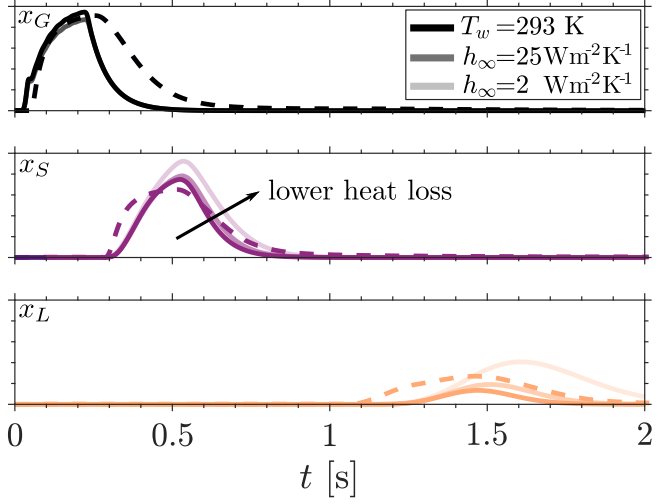

Figure 11: Time series of the centreline entropic perturbations $(\xi=0)$ : (a) comparison between experiments using thermocouples (TC) (- - ) and numerical results using URANS for test cases 1, 3,6 and 8 at $x_{G}=0.04 \mathrm{~m}(-), x_{S}=0.4 \mathrm{~m} \mathrm{(-)}$ and $x_{L}=1.4 \mathrm{~m} \mathrm{(-)}$ downstream of the heating grid; (b) comparison between TC and URANS results for case 1 with LITGS $(\bullet$ results phase-averaged from 30 shots [17]; (c) comparison between TC and URANS results for case 1 using the heat transfer boundary condition $\left(-h_{\infty}=25 \mathrm{~W} \mathrm{~m}^{-2} \mathrm{~K}^{-1}\right.$ and $\left.-h_{\infty}=2 \mathrm{~W} \mathrm{~m}^{-2} \mathrm{~K}^{-1}\right)$.

The time at which the entropy wave arrives at the probe location is independent of the temperature boundary condition. However, when the one-dimensional heat transfer boundary condition is used, the peak entropic perturbation increases significantly, both at $x_{S}$ and $x_{L}$. When the thermal flux at the wall is low (such as with the one-dimensional heat transfer boundary condition, in comparison to the isothermal wall condition), temperature fluctuations remain large in the boundary layer. Since convection of thermal fluctuations is lower in the boundary layer due to lower mean velocity, the radial temperature gradient between the walls and the centreline remains low in the back end of the temperature spot, leading to reduced thermal diffusion to the walls. This explains the larger centreline temperature fluctuations at the trailing edge of the entropic wave shown in Fig. 11 (c).

From this, we can conclude that, although the entropic fluctuations are relatively small $(\sigma \approx 0.1)$, the results downstream of the heating grid are highly sensitive to the temperature boundary condition at the wall. 


\section{Comparison of scalar perturbations}

The transport of the inhomogeneities can be explained by appropriately normalising the results with equal centreline velocities. In this study, the results are shifted in time relative to the $x_{2}$ probe location for the gas injection cases and the $x_{S}$ probe location for the heat addition case. This allows us to compare the changes in wave signature as they propagate downstream of the duct by overlapping them. The variables are normalised by the bulk convective time, $\tau_{c}=L_{c} / \bar{U}$ :

$$
\zeta=\left(t-\frac{z_{j}-x_{2}}{U_{c l}}\right) \tau_{c}^{-1}, \quad \eta=\left(t-\frac{z_{j}-x_{S}}{U_{c l}}\right) \tau_{c}^{-1},
$$

where $U_{c l} \approx 1.1 \mathrm{~m} \mathrm{~s}^{-1}$ is the centreline velocity extracted from the URANS simulations upstream of the injection, $L_{c}$ is the convective length (distance from the perturbation location to the outlet as shown in Fig. 1), and $j$ is a counter for the different probe locations. The bulk convective time is $\tau_{c}=0.6 \mathrm{~s}$ for the compositional cases and $\tau_{c}=1.6 \mathrm{~s}$ for the entropic case.

Figure 12 shows the results of this analysis using the probe volume-averaged results for the compositional cases. In the case of argon, the profiles can be entirely accounted for by the normalisation procedure. There are minor diffusive dispersion effects visible, which seem to flatten and spread the trailing edge spike seen in the first three probe locations. However, since convection is the main transport mechanism
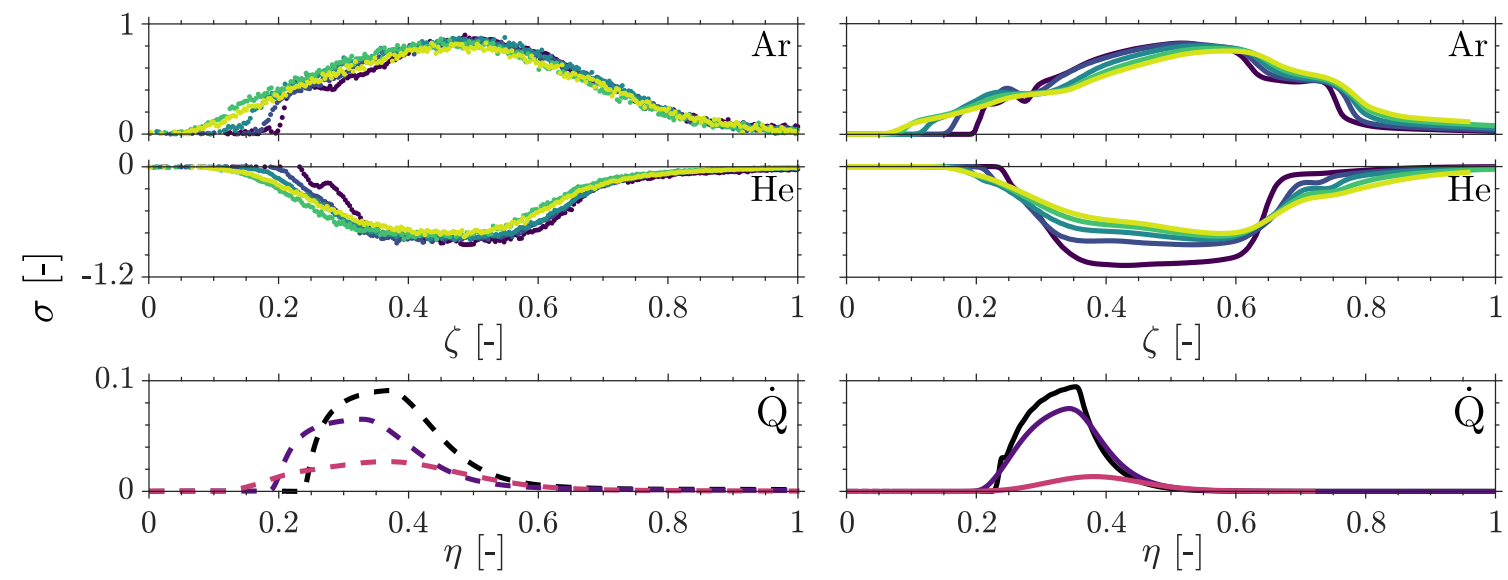

Figure 12: Centreline entropic perturbations as a function of time (shifted for each probe location and normalised by the respective bulk convective time). Comparison between experiments using LITGS (•) for the gas injection cases and thermocouples (-- -) for the heat addition (left) and numerical results using URANS (-) (right). For heat addition, case $1\left(\bar{U}=0.88 \mathrm{~m} \mathrm{~s}^{-1}\right)$ is shown. 
and species are conserved, the maximum perturbation amplitude remains constant. This also holds true for helium's experimental data, and is in contrast with the heat addition case, where significant thermal diffusion and heat loss to the wall means that the original perturbation decays quickly.

\section{Conclusions}

In this work, URANS calculations are undertaken to model the mixing of unsteady entropic and compositional waves in an open-ended flow duct. The computations are compared to experimental results obtained using both intrusive and non-intrusive techniques.

For the gas injection cases, argon produces a peak compositional perturbation on the $x-z$ mid-plane five times larger than that of helium. This is due to the low molecular mass of helium and the lower injection mass flow rate. Despite the lower injection mass flow rate, helium produces a larger absolute entropic disturbance owing to the considerable specific entropy difference between air and helium. For the heat addition cases, the absolute entropic disturbance generated is an order of magnitude smaller than that of argon and helium.

Despite the fact that the flow investigated is in the transitional regime, all scalars convect in similar ways and are reproduced well. For the comparison of the gas injection results, three sampling methodologies have been studied in the analysis of the numerical data: point, probe volume-averaged and cross-sectional area-averaged. For the injection of argon, agreement is found at all probe locations using all three sampling methodologies. The peak disturbance amplitude at the first probe for helium is well captured by the cross-section average value, but is overpredicted by the point and probe-volume averaged results. Despite this, good agreement is found for helium at the probes further downstream. For the heat addition cases, although the centreline temperature perturbations are small, it is found for the present system that heat transfer to the wall needs to be accounted for. The results at downstream locations lag behind the experiments in terms of arrival time for both perturbations, suggesting that the centreline velocity is underpredicted in the numerical simulations. However, no velocity measurements are currently available for comparison.

Future work will involve running fully compressible simulations with appropriate acoustic boundary conditions and nozzle geometries to resolve the direct and indirect noise contributions. This will directly yield the transfer function between an entropic or compositional wave, and the subsequent acoustic waves generated. 


\section{Acknowledgements}

Jocelino Rodrigues is supported by the Qualcomm European Research Studentship and the EPSRC DTA Studentship (University of Cambridge). The additional support from Rolls-Royce plc is also acknowledged. Thanks are given to Francesca De Domenico for providing the experimental data and discussing theoretical details, as well as to James Massey and Lee Weller for the fruitful conversations.

\section{References}

[1] A. P. Dowling, Y. Mahmoudi, Combustion noise, Proceedings of the Combustion Institute 35 (1) (2015) 65-100.

[2] C. S. Goh, A. S. Morgans, The Influence of Entropy Waves on the Thermoacoustic Stability of a Model Combustor, Combustion Science and Technology 185 (2) (2013) 249-268.

[3] W. Polifke, C. O. Paschereit, K. Paschereit, Constructive and Destructive Interference of Acoustic and Entropy Waves in a Premixed Combustor with a Choked Exit, The International Journal of Acoustics and Vibration 6 (3) (2001) $135-146$.

[4] L. Magri, On indirect noise in multicomponent nozzle flows, Journal of Fluid Mechanics 828 (R2).

[5] A. Giusti, L. Magri, M. Zedda, Flow Inhomogeneities in a Realistic Aeronautical Gas-Turbine Combustor: Formation, Evolution, and Indirect Noise, Journal of Engineering for Gas Turbines and Power 141 (1) (2019) 011502.

[6] F. Bake, U. Michel, I. Roehle, Investigation of Entropy Noise in Aero-Engine Combustors, Journal of Engineering for Gas Turbines and Power 129 (2) (2007) $370-376$.

[7] F. Bake, U. Michel, I. Roehle, Fundamental Mechanism of Entropy Noise in Aero-Engines: Experimental Investigation, in: Volume 6: Turbo Expo 2007, Parts A and B, vol. 130, ASME, 1445-1453, 2007.

[8] F. Bake, C. Richter, B. Mühlbauer, N. Kings, I. Röhle, F. Thiele, B. Noll, The Entropy Wave Generator (EWG): A reference case on entropy noise, Journal of Sound and Vibration 326 (3-5) (2009) 574-598. 
[9] N. Kings, F. Bake, Indirect Combustion Noise: Noise Generation by Accelerated Vorticity in a Nozzle Flow, International Journal of Spray and Combustion Dynamics 2 (3) (2010) 253-266.

[19] B. Mühlbauer, A. Widenhorn, M. Liu, B. Noll, M. Aigner, Fundamental mechanism of entropy noise in aero-engines: numerical simulation, Proceedings of ASME Turbo Expo 2007: Power for Land, Sea and Air (2007) 1-10. 
[20] B. Mühlbauer, B. Noll, M. Aigner, Numerical Investigation of the Fundamental Mechanism for Entropy Noise Generation in Aero-Engines, Acta Acustica united with Acustica 95 (3) (2009) 470-478.

[21] J.-M. Lourier, A. Huber, B. Noll, M. Aigner, Numerical Analysis of Indirect Combustion Noise Generation Within a Subsonic Nozzle, AIAA Journal 52 (10) (2014) 2114-2126.

[22] M. Leyko, F. Nicoud, S. Moreau, T. Poinsot, Numerical and analytical investigation of the indirect combustion noise in a nozzle, Comptes Rendus Mécanique 337 (6-7) (2009) 415-425.

[23] M. Leyko, S. Moreau, F. Nicoud, T. Poinsot, Numerical and analytical modelling of entropy noise in a supersonic nozzle with a shock, Journal of Sound and Vibration 330 (16) (2011) 3944-3958.

[24] I. Durán, S. Moreau, T. Poinsot, Analytical and Numerical Study of Combustion Noise Through a Subsonic Nozzle, AIAA Journal 51 (1) (2013) 42-52.

[25] C. Becerril, S. Moreau, M. Bauerheim, L. Gicquel, T. Poinsot, Numerical investigation of combustion noise: The Entropy Wave Generator, in: 22nd AIAA/CEAS Aeroacoustics Conference, American Institute of Aeronautics and Astronautics, Reston, Virginia, 1-18, 2016.

[26] S. Moreau, C. Becerril, L. Gicquel, Large-Eddy-simulation prediction of indirect combustion noise in the entropy wave generator experiment, International Journal of Spray and Combustion Dynamics 10 (2) (2018) 154-168.

[27] T. Sattelmayer, Influence of the Combustor Aerodynamics on Combustion Instabilities From Equivalence Ratio Fluctuations, Journal of Engineering for Gas Turbines and Power 125 (1) (2003) 11-19.

[28] A. S. Morgans, C. S. Goh, J. A. Dahan, The dissipation and shear dispersion of entropy waves in combustor thermoacoustics, Journal of Fluid Mechanics $733(\mathrm{R} 2)$.

[29] A. Giusti, N. A. Worth, E. Mastorakos, A. P. Dowling, Experimental and Numerical Investigation into the Propagation of Entropy Waves, AIAA Journal 55 (2) (2017) 446-458.

[30] T. F. Fric, A. Roshko, Vortical structure in the wake of a transverse jet, Journal of Fluid Mechanics 279 (1) (1994) 1-47. 
[31] A. T. Hsu, G. He, Y. Guo, Unsteady Simulation of a Jet-in-crossflow, International Journal of Computational Fluid Dynamics 14 (1) (2000) 41-53.

[32] B. Launder, D. Spalding, The numerical computation of turbulent flows, Computer Methods in Applied Mechanics and Engineering 3 (2) (1974) 269-289.

[33] I. Tkatchenko, N. Kornev, S. Jahnke, G. Steffen, E. Hassel, Performances of LES and RANS Models for Simulation of Complex Flows in a Coaxial Jet Mixer, Flow, Turbulence and Combustion 78 (2) (2007) 111-127.

[34] E. M. Ivanova, B. E. Noll, M. Aigner, Computational Modeling of Turbulent Mixing of a Transverse Jet, Journal of Engineering for Gas Turbines and Power $133(2)$.

[35] F. C. C. Galeazzo, G. Donnert, C. Cárdenas, J. Sedlmaier, P. Habisreuther, N. Zarzalis, C. Beck, W. Krebs, Computational modeling of turbulent mixing in a jet in crossflow, International Journal of Heat and Fluid Flow 41 (2013) $55-65$.

[36] E. O. Rolland, Sound produced by entropic and compositional inhomogeneities, Ph.D. thesis, 2018.

[37] N. Cumpsty, Jet engine combustion noise: Pressure, entropy and vorticity perturbations produced by unsteady combustion or heat addition, Journal of Sound and Vibration 66 (4) (1979) 527-544.

[38] M. Leyko, F. Nicoud, T. Poinsot, Comparison of Direct and Indirect Combustion Noise Mechanisms in a Model Combustor, AIAA Journal 47 (11) (2009) 2709 2716 .

[39] W. H. Bragg, W. L. Bragg, The Reflection of X-rays by Crystals, Proceedings of the Royal Society A: Mathematical, Physical and Engineering Sciences 88 (605) (1913) 428-438.

[40] S. B. Pope, Turbulent Flows, Cambridge University Press, 2000.

[41] S. Chapman, T. G. Cowling, The Mathematical Theory of Non-Uniform Gases, Cambridge University Press, 1970.

[42] Y. Tominaga, T. Stathopoulos, Turbulent Schmidt numbers for CFD analysis with various types of flowfield, Atmospheric Environment 41 (37) (2007) 80918099 . 
[43] F. R. Menter, Two-Equation Eddy-Viscosity Turbulence Models for Engineering Applications, AIAA journal 32 (8) (1994) 1598-1605.

[44] D. G. Goodwin, H. K. Moffat, R. L. Speth, Cantera: An object-oriented software toolkit for chemical kinetics, thermodynamics, and transport processes (Version 2.3.0), 2017.

[45] A. Malhotra, S. Kang, Turbulent Prandtl number in circular pipes, International Journal of Heat and Mass Transfer 27 (11) (1984) 2158-2161.

[46] D. McEligot, M. Taylor, The turbulent Prandtl number in the near-wall region for low-Prandtl-number gas mixtures, International Journal of Heat and Mass Transfer 39 (6) (1996) 1287-1295.

[47] T. Bergman, A. Lavine, F. Incropera, D. DeWitt, Fundamentals of Heat and Mass Transfer, Wiley, 2011.

[48] R. Hilpert, Wärmeabgabe von geheizten Drähten und Rohren im Luftstrom, Forschung auf dem Gebiete des Ingenieurwesens 4 (5) (1933) 215-224. 